\title{
Synthesis and physical-chemical properties of (3-benzyl-8-propylxanthin-7-yl)acetohydrazide derivatives and their evaluation for antimicrobial and diuretic activities
}

\author{
K. V. Aleksandrova (DA,F, Ye. K. Mykhalchenko iD*B,C, O. S. Shkoda DD,E, D. A. Vasyliev IDD,E \\ Zaporizhzhia State Medical University, Ukraine
}

A - research concept and design; B - collection and/or assembly of data; C - data analysis and interpretation; D - writing the article;

$\mathrm{E}$ - critical revision of the article; $\mathrm{F}$ - final approval of the article

One of the most important tasks of our indigenous pharmaceutical science is the necessity for new medicines because existing drugs are characterized by various side effects, resistance, high toxicity, and so on. New bioactive molecule synthesis utilizes substances of natural origin as well as chemically modified ones. Thus, the researcher's attention is mainly focused on 3-,7-,8-substituted derivatives of the natural heterocyclic xanthine system, which possess a wide range of pharmacological action. Synthesis of a novel of (3-benzyl-8propylxanthin-7-yl)acetohydrazides with antimicrobial and diuretic activities described in the paper.

The aim of this work is to develop efficient methods for synthesis of (3-benzyl-8-propylxanthin-7-yl)acetohydrazide derivatives, and to study their physical-chemical properties.

Materials and methods. Two-hour boiling of propyl 2-(3-benzyl-8-propylxanthine-7-yl)acetate by excess hydrazine hydrate in propan-2-ol medium have yielded the key the key intermediate 2-(3-benzyl-8-propylxanthine-7-yl)acetohydrazide. Further transformation of the latter has led to formation of corresponding acetohydrazide derivatives achieved by the reaction with aliphatic, aromatic, heterocyclic aldehydes, and ketones. The structure and the relative configuration of the synthesized compounds were elucidated by analyzing their physical-chemical data.

Results. The synthesis and optimization of reaction conditions of (3-benzyl-8-propylxanthin-7-yl)acetohydrazide derivatives were conducted. The identification of all synthesized compounds was aided by various physical-chemical methods (thin layer chromatography, elemental analysis, IR, and ${ }^{1} \mathrm{H}$ NMR spectroscopy).

Conclusions. As a result of synthetic research the preparative synthesis method of (3-benzyl-8-propylxanthin-7-yl)acetohydrazide derivatives possessing antimicrobic, and diuretic activities was developed.

Key words: 3-benzylxanthine, acetohydrazide, physicochemical properties.

Current issues in pharmacy and medicine: science and practice 2021; 14 (1), 17-22

Синтез і фрізико-хімічні властивості іліденпохідних гідразиду 3-бензил-8-пропілксантиніл-7-ацетатної кислоти, що мають протимікробні та діуретичні властивості

\section{К. В. Александрова, Є. К. Михальченко, О.С.Шкода, Д. А. Васильєв}

Створення нових лікарських препаратів - одне з найважливіших завдань вітчизняної фрармацевтичної науки. Це зумовлено наявністю у препаратів небажаних побічних ефектів, набуттям резистентності, високою токсичністю тощо. Для створення біоактивних молекул застосовують і речовини природного походження, і нові синтезовані сполуки та продукти їхньої хімічної модифікації. У цьому аспекті увагу дослідників привертають 3-,7-,8-заміщені похідні природної гетероциклічної системи ксантину, що мають широкий спектр фармакологічної дії. Наведено синтез неописаних у фаховій літературі іліденгідразидів 3-бензил-8-пропілксантин-7-іл ацетатної кислоти, які характеризуються протимікробними та діуретичними властивостями.

Мета роботи - розроблення методів одержання іліденпохідних 3-бензил-8-пропілксантиніл-7-ацетатної кислоти та вивчення фізико-хімічних властивостей цих сполук.

Матеріали та методи. Як вихідну сполуку використали гідразид 3-бензил-8-пропілксантиніл-7-ацетатної кислоти, одержаний раніше двогодинним кип'ятінням н-пропілового естеру 3-бензил-8-пропілксантиніл-7-ацетатної кислоти з надлишком гідразин гідрату в середовищі пропан-2-олу. Надалі вивчили реакцію гідразиду 3-бензил-8-пропілксантиніл-7-ацетатної кислоти з аліфатичними, ароматичними, гетероциклічними альдегідами та кетонами, в результаті отримали відповідні іліденпохідні. Будова та індивідуальність синтезованих сполук підтверджена комплексом фізико-хімічних досліджень.

ARTICLE

INFO

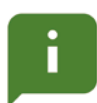

http:l/pharmed.

zsmu.edu.ualarticle/ view/226742
UDC 547.854.4'792'572.3'211.024.03/.04.057

DOI: $10.14739 / 2409-2932.2021 .1 .226742$

Current issues in pharmacy and medicine: science and practice $2021 ; 14$ (1), 17-22

Key words: 3-benzylxanthine, acetohydrazide, physicochemical properties.

*E-mail: ekm0989@gmail.com

Received: 26.10.2020 // Revised: 11.11.2020 // Accepted: 17.11.2020 
Результати. Здійснили синтез і визначили оптимальні умови перебігу реакцій одержання іліденпохідних 3-бензил-8-пропілксантиніл-7-ацетатної кислоти. За допомогою фрізико-хімічних методів дослідження (хроматографія в тонкому шарі сорбенту, елементний аналіз, ІЧ- та ${ }^{1} \mathrm{H}$ ЯМР-спектроскопія) встановили структуру та індивідуальність нових синтезованих сполук.

Висновки. У результаті синтетичного дослідження опрацювали препаративну методику синтезу іліденгідразидів 3-бензил-8-пропілксантиніл-7-ацетатної кислоти, які мають протимікробні та діуретичні властивості.

Ключові слова: 3-бензилксантин, іліденгідразиди, фрізико-хімічні властивості.

Актуальні питання фрармацевтичної і медичної науки та практики. 2021. Т. 14, № 1(35). C. 17-22

Синтез и физико-химические свойства илиденпроизводных гидразида 3-бензил-8-пропилксантинил-7-ацетатной кислоты, которые проявляют противомикробные и диуретические свойства

\section{Е. В. Александрова, Е. К. Михальченко, А. С. Шкода, Д. А. Васильев}

Создание новых лекарственных препаратов - одна из важнейших задач отечественной фрармацевтической науки. Это объясняется наличием у существующих препаратов нежелательных побочных эффектов, приобретением резистентности, высокой токсичностью и т. д. Для создания биоактивных молекул применяются и вещества природного происхождения, и новосинтезированные соединения и продукты их химической модификации. В этом плане внимание исследователей привлекают 3,7,8-замещенные производные природной гетероциклической системы ксантина, проявляющие широкий спектр фрармакологической активности. Представлен синтез неописанныХ в специализированной литературе илиденгидразидов 3-бензил-8-пропилксантинил-7-ацетатной кислоты, которые проявляют противомикробную и диуретическую активности.

Цель работы - разработка методов получения илиденпроизводных 3-бензил-8-пропилксантинил-7-ацетатной кислоты и изучение фризико-химических свойств этих соединений.

Материалы и методы. В качестве исходного соединения использован гидразид 3-бензил-8-пропилксантинил-7-ацетатной кислоты, полученный ранее двухчасовым кипячением н-пропилового эфира 3-бензил-8-пропилксантинил-7-ацетатной кислоты с избытком гидразингидрата в среде пропанола-2. В дальнейшем изучена реакция гидразида 3-бензил-8-пропилксантинил-7-ацетатной кислоты с алифатическими, ароматическими, гетероциклическими альдегидами и кетонами, в результате которой получены соответствующие илиденпроизводные. Строение и индивидуальность синтезированных соединений подтверждена комплексом фризико-химических исследований.

Результаты. Осуществлен синтез и определены оптимальные условия протекания реакций получения илиденпроизводных 3-бензил-8-пропилксантинил-7-ацетатной кислоты. С помощью фризико-химических методов исследования (хроматография в тонком слое сорбента, элементный анализ, ИК и ${ }^{1} \mathrm{H}$ ЯМР-спектроскопия) установлены структура и индивидуальность новосинтезированных соединений.

Выводы. В результате синтетического исследования разработана препаративная методика синтеза илиденгидразидов 3-бензил-8-пропилксантинил-7-ацетатной кислоты, которые проявляют противомикробную и диуретическую активности.

Ключевые слова: 3-бензилксантин, илиденгидразиды, фризико-химические свойства.

Актуальные вопросы фрармацевтической и медицинской науки и практики. 2021. Т. 14, № 1(35). С. 17-22

In recent years, xanthine derivatives have attracted researchers' attention not only by their frequent occurrence in nature but also by their diverse pharmacological activities [1-5].

Xanthines, as important members of the purine family, are considered as key intermediates in the metabolism of nucleobases, because metabolic interconversions of adenine and guanine result in their formation [6,7]. In addition, xanthine, and its derivatives act as precursors in the synthesis of GMP, GDP, and GTP through the salvage pathways within cells [8]. Due to the aforementioned reasons, it is important to explore the use of xanthine derivatives in developing novel synthetic methods. Our interest is in finding effective acetohydrazides of (3-R-xanthine-7(8)-yl)alkanoic acids, and their structural analogs, because such compounds have been reported in the literature by their diverse biological activities.

\section{Aim}

Considering the abovementioned evidence, we made endeavors to synthesize a set of novel (3-benzyl-8-propylxanthin-7-yl) acetohydrazide derivatives and to study their physical-chemical properties.

\section{Materials and methods}

Unless otherwise indicated, reagents and solvents were purchased from commercial suppliers and used without further purification.

The starting material of (3-benzyl-8-propylxanthin-7-yl) acetohydrazide (1) was synthesized by boiling of n-propyl ester with hydrazine hydrate excess in propan-2-ol medium (Fig. 1).

The procedure for the synthesis of (3-benzyl-8-propylxanthin-7-yl)acetohydrazide (1). A suspension of $3.84 \mathrm{~g}(0.01$ mol) of propyl 2-(3-benzyl-2,6-dioxo-8-propyl-1,2,3,6-tetrahydro-7H-purin-7-yl)acetate was boiled under reflux in $30 \mathrm{ml}$ of propan-2-ol. This mixture was heated for $15 \mathrm{~min}$ to form a clear solution. Then $5 \mathrm{ml}$ of hydrazine hydrate was added and boiled for 45 minutes. After cooling the mixture to room temperature, the solution was poured into $100 \mathrm{ml}$ of water. The precipitate was filtered, washed with water, and dried at $80-85{ }^{\circ} \mathrm{C}$. Yield $87 \%$.

The chemical structure of (3-benzyl-8-propylxanthin-7-yl) acetohydrazide (1) was determined by ${ }^{1} \mathrm{H}$ NMR spectroscopy. The ${ }^{1} \mathrm{H}$ NMR spectrum was revealed the appearance of a downfield singlet at $\delta 11.16 \mathrm{ppm}$, which confirms the uracil 

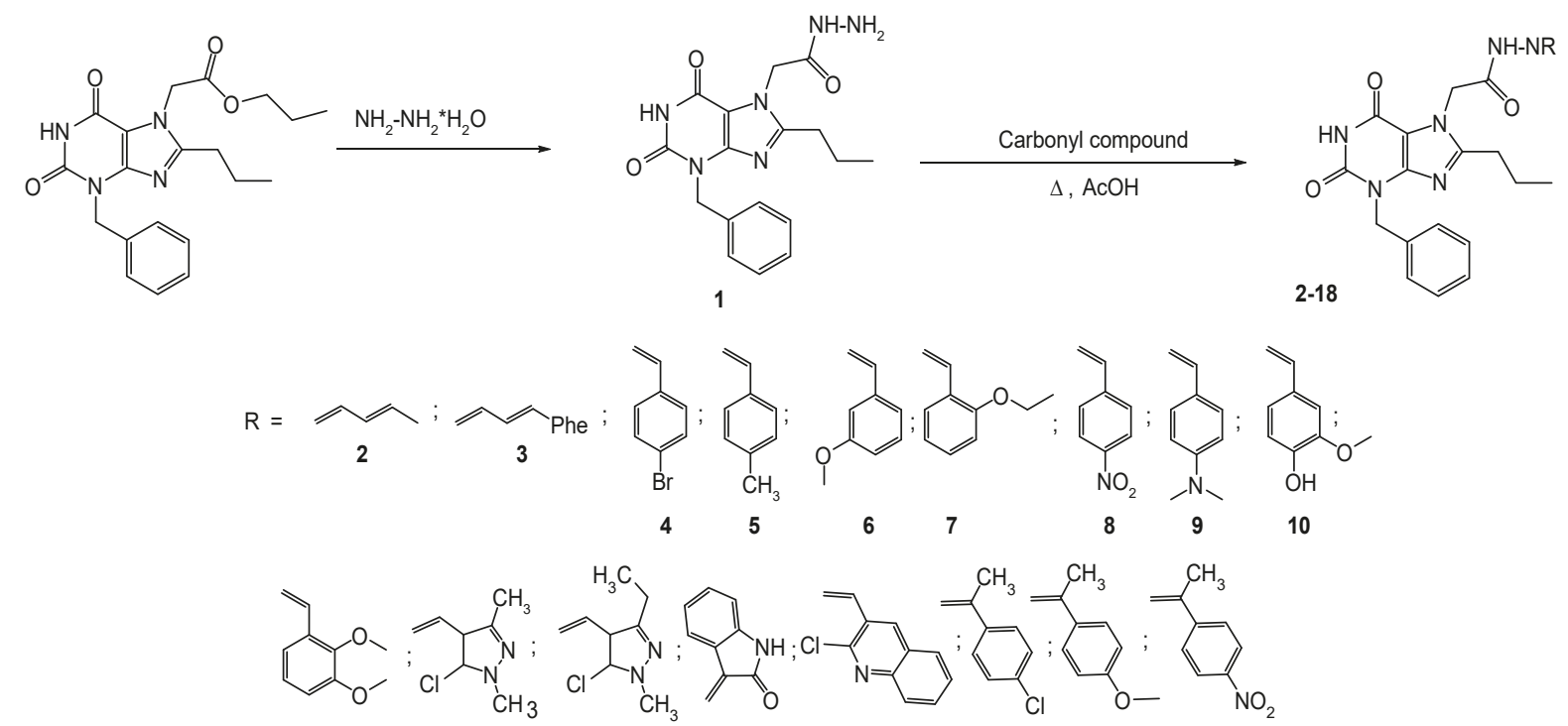

11

13

14

15

$16 \quad 17$

18

Fig. 1. Synthesis of (3-benzyl-8-propylxanthin-7-yl)acetohydrazide and its derivatives (1-18).

core of the investigated molecule. Broad singlet signal at $\delta$ $9.34 \mathrm{ppm}$ was represented NH-group of hydrazide residue, whereas broad signal assigned for two protons of $\mathrm{NH}_{2}$-group at $\delta 4.50-4.32 \mathrm{ppm}$. Signals of aromatic protons, methylene and methyl groups were recorded without anomalies in intensities, chemical shifts and cleavage at $\delta 7.27-7.10 \mathrm{ppm}(\mathrm{m}, 5 \mathrm{H}, \mathrm{Ar}-\mathrm{H})$; $5.08 \mathrm{ppm}\left(\mathrm{s}, 2 \mathrm{H}, \mathrm{N}^{3} \mathrm{CH}_{2}\right) ; 4.98 \mathrm{ppm}\left(\mathrm{s}, 2 \mathrm{H} . \mathrm{N}^{7} \mathrm{CH}_{2}\right) ; 2.59 \mathrm{ppm}$ (t, $\left.2 \mathrm{H}, J=7.41 \mathrm{~Hz}, \mathrm{C}^{8} \mathrm{CH}_{2}\right) ; 1,62 \mathrm{ppm}(\mathrm{m}, 2 \mathrm{H}, J=7.42 \mathrm{~Hz}$, $\left.\mathrm{C}^{8} \mathrm{C}-\mathrm{CH}_{2}\right) ; 0,89 \mathrm{ppm}\left(\mathrm{t}, 3 \mathrm{H}, J=7.38 \mathrm{~Hz}, \mathrm{C}^{8} \mathrm{C}-\mathrm{C}-\mathrm{CH}_{3}\right)$.

Thereafter, the reaction of (3-benzyl-8-propylxanthin-7-yl)acetohydrazide (1) with aliphatic, aromatic, heterocyclic carbonyl compounds was studied and the corresponding acetohydrazide derivatives (2-18) were obtained. The interaction of equimolar amounts of reactants was preceded in boiling water-dioxane medium for 30-40 minutes with acetic acid as a catalyst (Fig. 1).

The study of physical-chemical properties of the synthesized compounds was performed according to the State Pharmacopoeia of Ukraine (ed. 1). The melting point was determined by capillary method (2.2.14) on the PTP(M) device.

Infrared spectra were recorded on a Bruker Alpha (Bruker, Germany) in 4000-400 $\mathrm{cm}^{-1}$ with ATR (direct material input). ${ }^{1} \mathrm{H}-\mathrm{NMR}$ spectra were recorded on Varian Mercury VX-200 spectrometer (Varian, USA) in DMSO- $\mathrm{d}_{6}$ with TMS as an internal standard. Elemental analysis was performed on a Elementar Vario L cube analyzer (Elementar Analysensysteme, Germany). Binary mobile phase of benzene and propan-2-ol in $10: 1$ and $1: 10$ was used for chromatography. Chromatographic spots were visualized by UV light (200-300 nm).

\section{Results}

Synthesis (3-benzyl-8-propylxanthin-7-yl)acetohydrazide and its derivatives (1-18) showed in Fig. 1.
General procedure for the synthesis of (3-benzyl-8propylxanthin-7-yl)acetohydrazide derivatives (2-18). To a solution of $3.56 \mathrm{~g}(0.01 \mathrm{~mol})$ of 1 in $70 \mathrm{ml}$ of aqueous dioxane (1:1) and heated to $50{ }^{\circ} \mathrm{C}$ was added $1 \mathrm{ml}$ of glacial acetic acid and $0.011 \mathrm{~mol}$ of the corresponding aldehyde or ketone. The mixture was heated under a reflux condenser for 15-25 minutes. After cooling, the resulting product was precipitated. The crude product was filtered, washed with water, and dried at $80-85^{\circ} \mathrm{C}$.

Compounds 2-18 were pale-yellow, yellow or orange amorphous substances, insoluble in water, ethanol, diethyl ether, acetone, and soluble in DMF.

The structure of the synthesized compounds was confirmed by elemental analysis data (Table 1), and by IR- and ${ }^{1} \mathrm{H}$ NMR spectroscopy (Tables 2, 3).

In the IR spectra of compounds 2-18 (Table 2): $v_{\max }(\mathrm{KBr}$, $\mathrm{cm}^{-1}$ ): 3620-3600 (N-H stretching), 2989-2887 (al. C-H asymmetrical and symmetrical stretching), 1729-1677, 1670-1630, 1597-1570 ( $\mathrm{C}=\mathrm{O}, \mathrm{C}=\mathrm{N}, \mathrm{C}=\mathrm{C}$ stretching).

The ${ }^{1} \mathrm{H}$ NMR spectra of compounds $2-18$ (Table 3 ) showed absence of the $\mathrm{NH}_{2}$-group protons of the hydrazide residue, and NH-groups signal of the ylidene hydrazide residue was appeared as downfield singlet at $\delta 12.08-11.08$ ppm. Signals of protons of methylidene groups were appeared as singlet at $\delta 8.61-7.85 \mathrm{ppm}$, protons of benzyl, propyl residue and uracil fragment of the molecule were recorded with the appropriate intensity and characteristic chemical shift.

Thus, ${ }^{1} \mathrm{H}$ NMR spectrum 2-(3-benzyl-8-propylxanthin-7-yl)- $N$ '-[4-bromobenzyliden] acetohydrazide (4) was revealed the appearance of three proton singlets of $\mathrm{NH}$ groups: a substituent in the 7-position of the xanthine bicycle (11.82 ppm) and uracil fragment (11.08 ppm) and $\mathrm{N}=\mathrm{CH}(7.98$ ppm). Aromatic protons of the 4-bromobenzylidene moiety 
Table 1. Physical-chemical characteristics of (3-benzyl-8-propylxanthin-7-yl)acetohydrazide derivatives (2-18)

\begin{tabular}{|c|c|c|c|c|c|c|c|c|c|c|}
\hline \multirow{2}{*}{ Comp. } & \multirow{2}{*}{$\mathrm{Mp} .{ }^{\circ} \mathrm{C}$} & \multirow{2}{*}{$R_{f}$} & \multicolumn{3}{|c|}{ Anal. calcd. \% } & \multirow{2}{*}{ Formula } & \multicolumn{3}{|c|}{ Found. $\%$} & \multirow{2}{*}{ Yield. \% } \\
\hline & & & C & H & N & & C & H & N & \\
\hline 1 & 3 & 4 & 5 & 6 & 7 & 8 & 9 & 10 & 11 & 12 \\
\hline 2 & $237-239$ & 0.78 & 61.71 & 5.91 & 2.54 & $\mathrm{C}_{21} \mathrm{H}_{24} \mathrm{~N}_{6} \mathrm{O}_{3}$ & 61.75 & 5.95 & 2.58 & 80 \\
\hline 3 & $222-225$ & 0.80 & 66.34 & 5.54 & 17.82 & $\mathrm{C}_{26} \mathrm{H}_{26} \mathrm{~N}_{6} \mathrm{O}_{3}$ & 66.37 & 5.57 & 17.86 & 82 \\
\hline 4 & $276-279$ & 0.56 & 55.04 & 4.39 & 16.02 & $\mathrm{C}_{24} \mathrm{H}_{23} \mathrm{BrN}_{6} \mathrm{O}_{3}$ & 55.08 & 4.43 & 16.06 & 83 \\
\hline 5 & $240-242$ & 0.54 & 65.45 & 5.68 & 18.29 & $\mathrm{C}_{25} \mathrm{H}_{26} \mathrm{~N}_{6} \mathrm{O}_{3}$ & 65.49 & 5.72 & 18.33 & 80 \\
\hline 6 & $233-235$ & 0.63 & 63.24 & 5.48 & 17.67 & $\mathrm{C}_{25} \mathrm{H}_{26} \mathrm{~N}_{6} \mathrm{O}_{4}$ & 63.28 & 5.52 & 17.71 & 82 \\
\hline 7 & 204-206 & 0.56 & 63.89 & 5.74 & 17.16 & $\mathrm{C}_{26} \mathrm{H}_{28} \mathrm{~N}_{6} \mathrm{O}_{4}$ & 63.92 & 5.78 & 17.20 & 86 \\
\hline 8 & $281-283$ & 0.80 & 58.85 & 4.70 & 19.98 & $\mathrm{C}_{24} \mathrm{H}_{23} \mathrm{~N}_{7} \mathrm{O}_{5}$ & 58.89 & 4.74 & 20.03 & 90 \\
\hline 9 & 292-295 & 0.68 & 64.00 & 5.96 & 20.7 & $\mathrm{C}_{26} \mathrm{H}_{29} \mathrm{~N}_{7} \mathrm{O}_{3}$ & 64.05 & 6.00 & 20.11 & 85 \\
\hline 10 & $244-246$ & 0.56 & 61.18 & 5.30 & 17.9 & $\mathrm{C}_{25} \mathrm{H}_{26} \mathrm{~N}_{6} \mathrm{O}_{5}$ & 61.22 & 5.34 & 17.13 & 84 \\
\hline 11 & $108-110$ & 0.72 & 61.85 & 5.55 & 16.62 & $\mathrm{C}_{26} \mathrm{H}_{28} \mathrm{~N}_{6} \mathrm{O}_{5}$ & 61.89 & 5.59 & 16.66 & 85 \\
\hline 12 & $267-270$ & 0.64 & 55.55 & 5.03 & 22.51 & $\mathrm{C}_{23} \mathrm{H}_{25} \mathrm{ClN}_{8} \mathrm{O}_{3}$ & 55.59 & 5.07 & 22.55 & 90 \\
\hline 13 & $261-263$ & 0.58 & 56.37 & 5.29 & 20.89 & $\mathrm{C}_{24} \mathrm{H}_{27} \mathrm{CIN}_{8} \mathrm{O}_{3}$ & 56.41 & 5.33 & 21.93 & 84 \\
\hline 14 & $255-257$ & 0.68 & 61.81 & 4.74 & 20.16 & $\mathrm{C}_{25} \mathrm{H}_{23} \mathrm{~N}_{7} \mathrm{O}_{4}$ & 61.85 & 4.78 & 20.20 & 80 \\
\hline 15 & $262-264$ & 0.66 & 61.15 & 4.52 & 18.46 & $\mathrm{C}_{27} \mathrm{H}_{24} \mathrm{ClN}_{7} \mathrm{O}_{3}$ & 61.19 & 4.56 & 18.50 & 82 \\
\hline 16 & $247-249$ & 0.54 & 60.87 & 5.7 & 17.01 & $\mathrm{C}_{25} \mathrm{H}_{25} \mathrm{ClN}_{6} \mathrm{O}_{3}$ & 60.91 & 5.11 & 17.05 & 86 \\
\hline 17 & $235-238$ & 0.72 & 63.88 & 5.74 & 17.16 & $\mathrm{C}_{26} \mathrm{H}_{28} \mathrm{~N}_{6} \mathrm{O}_{4}$ & 63.92 & 5.78 & 17.20 & 81 \\
\hline 18 & 280-282 & 0.68 & 59.59 & 4.96 & 19.43 & $\mathrm{C}_{25} \mathrm{H}_{25} \mathrm{~N}_{7} \mathrm{O}_{5}$ & 59.63 & 5.00 & 19.47 & 84 \\
\hline
\end{tabular}

Table 2. IR-spectra of (3-benzyl-8-propylxanthin-7-yl)acetohydrazide derivatives (2-18)

\begin{tabular}{|c|c|c|c|c|c|c|}
\hline \multirow{2}{*}{ Comp. } & \multicolumn{6}{|l|}{$\mathrm{u}, \mathrm{cm}^{-1}$} \\
\hline & NH & Ar-H & $C=0$ & $\mathrm{C}=\mathrm{N}$ & $C=C$ & Other \\
\hline 2 & $3320 ; 3170$ & 3032 & $1708 ; 1693$ & 1670 & 1580 & 2930 \\
\hline 3 & $3290 ; 3120$ & 3040 & $1710 ; 1680$ & 1640 & 1592 & 2920 \\
\hline 4 & $3260 ; 3150$ & 2987 & $1720 ; 1691$ & 1654 & 1589 & $2910 ; 717$ \\
\hline 5 & $3250 ; 3160$ & 3061 & $1710 ; 1680$ & 1630 & 1570 & $2980 ; 730$ \\
\hline 6 & $3290 ; 3130$ & 3047 & $1719 ; 1700$ & 1642 & 1576 & 2967 \\
\hline 7 & $3250 ; 3120$ & 3010 & $1700 ; 1690$ & 1667 & 1584 & 2887 \\
\hline 8 & $3280 ; 3160$ & 3030 & $1700 ; 1679$ & 1660 & 1592 & $2960 ; 1251$ \\
\hline 9 & $3260 ; 3130$ & 2997 & $1703 ; 1689$ & 1642 & 1583 & 2910 \\
\hline 10 & $3290 ; 3150$ & 3020 & $1700 ; 1678$ & 1640 & 1574 & $3600 ; 754$ \\
\hline 11 & $3310 ; 3150$ & 3035 & $1723 ; 1680$ & 1655 & 1595 & $2989 ; 715$ \\
\hline 12 & $3260 ; 3110$ & 3020 & $1710 ; 1696$ & 1633 & 1580 & $2971 ; 788$ \\
\hline 13 & $3270 ; 3140$ & 3010 & $1720 ; 1690$ & 1670 & 1590 & 2950 \\
\hline 14 & $3260 ; 3139$ & 3033 & $1700 ; 1680$ & 1660 & 1560 & 2940 \\
\hline 15 & $3260 ; 3109$ & 3080 & $1713 ; 1679$ & 1658 & 1597 & 2987 \\
\hline 16 & $3270 ; 3150$ & 3030 & $1715 ; 1679$ & 1666 & 1574 & 2910 \\
\hline 17 & $3300 ; 3180$ & 3050 & $1720 ; 1680$ & 1660 & 1590 & 2980 \\
\hline 18 & $3250 ; 3140$ & 3020 & $1710 ; 1692$ & 1649 & 1587 & 3610 \\
\hline
\end{tabular}


Table 3. ${ }^{1} \mathrm{H}$ NMR-spectra of (3-benzyl-8-propylxanthin-7-yl)acetohydrazide derivatives (2-18)

\begin{tabular}{|c|c|c|c|c|c|c|c|}
\hline \multirow[b]{2}{*}{ Comp. } & \multicolumn{7}{|l|}{ б. ppm } \\
\hline & $\begin{array}{l}\text { CONH } \\
(s, 1 \mathrm{H})\end{array}$ & $\begin{array}{l}N^{1} H \\
(s, 1 H)\end{array}$ & $\begin{array}{l}\mathrm{N}=\mathrm{CH} \\
(\mathrm{s}, 1 \mathrm{H})\end{array}$ & Ar-H & $\mathrm{CH}_{2}$ & $\mathrm{CH}_{3}$ & Others \\
\hline 2 & 11.71 & 11.17 & 7.89 & 7.35-7.18 (m, 5H) & $\begin{array}{l}5.46(\mathrm{~s}, 2 \mathrm{H}) ; 5.11(\mathrm{~s}, 2 \mathrm{H}) ; \\
2.71-2.58(\mathrm{t}, 2 \mathrm{H} \mathrm{J}=7.46 \mathrm{~Hz}) ; \\
1.76-1.61(\mathrm{~m}, 2 \mathrm{H} \mathrm{J}=7.44 \mathrm{~Hz})\end{array}$ & $\begin{array}{l}1.86(\mathrm{~d}, 3 \mathrm{H} \mathrm{J}=7.32 \mathrm{~Hz}) \\
0.92(\mathrm{t}, 3 \mathrm{H} \mathrm{J}=7.42 \mathrm{~Hz})\end{array}$ & - \\
\hline 3 & 11.67 & 11.19 & 7.86 & $\begin{array}{l}\text { 7.62-7.49 (m, 3H); } \\
\text { 7.46-7.14 (m, 5H); } \\
\text { 7.04-6.96 (d, 2H) }\end{array}$ & $\begin{array}{l}5.48(\mathrm{~s}, 2 \mathrm{H}) ; 5.09(\mathrm{~s}, 2 \mathrm{H}) \\
2.69-2.59(\mathrm{t}, 2 \mathrm{H} \mathrm{J}=7.36 \mathrm{~Hz}) ; \\
1.74-1.60(\mathrm{~m}, 2 \mathrm{H} \mathrm{J}=7.35 \mathrm{~Hz})\end{array}$ & $0.91(\mathrm{t}, 3 \mathrm{H} \mathrm{J}=7.33 \mathrm{~Hz})$ & $\begin{array}{l}7.09(\mathrm{~d}, 1 \mathrm{H})-\mathrm{CH} \\
6.85(\mathrm{~d}, 1 \mathrm{H})-\mathrm{CH}\end{array}$ \\
\hline 4 & 11.82 & 11.08 & 7.98 & $\begin{array}{l}\text { 7.71-7.61 (m, 4H); } \\
\text { 7.27-7.19 (m, 5H) }\end{array}$ & $\begin{array}{l}5.48(\mathrm{~s}, 2 \mathrm{H}) ; 5.06(\mathrm{~s}, 2 \mathrm{H}) ; \\
2.67-2.58(\mathrm{t}, 2 \mathrm{H} \mathrm{J}=7.44 \mathrm{~Hz}) ; \\
1.74-1.58(\mathrm{~m}, 2 \mathrm{H} \mathrm{J}=7.41 \mathrm{~Hz})\end{array}$ & $0.92(\mathrm{t}, 3 \mathrm{H} \mathrm{J}=7.42 \mathrm{~Hz})$ & - \\
\hline 5 & 11.81 & 11.12 & 7.99 & $\begin{array}{l}\text { 7.80-7.67 (m, 2H); } \\
\text { 7.37-7.18 (m, 7H) }\end{array}$ & $\begin{array}{l}5.49(\mathrm{~s}, 2 \mathrm{H}) ; 5.07(\mathrm{~s}, 2 \mathrm{H}) ; \\
2.66-2.56(\mathrm{t}, 2 \mathrm{H} \mathrm{J}=7.37 \mathrm{~Hz}) ; \\
1.72-1.59(\mathrm{~m}, 2 \mathrm{H} \mathrm{J}=7.36 \mathrm{~Hz})\end{array}$ & $\begin{array}{l}2.43(\mathrm{~s}, 3 \mathrm{H}) ; \\
0.91(\mathrm{t}, 3 \mathrm{H} \mathrm{J}=7.32 \mathrm{~Hz})\end{array}$ & - \\
\hline 6 & 11.66 & 11.08 & 8.35 & $\begin{array}{l}\text { 7.44-7.24 (m, 6H); } \\
\text { 7.11-6.92 (m, 3H) }\end{array}$ & $\begin{array}{l}5.44(\mathrm{~s}, 2 \mathrm{H}) ; 5.06(\mathrm{~s}, 2 \mathrm{H}) ; \\
2.67-2.55(\mathrm{t}, 2 \mathrm{H} \mathrm{J}=7.35 \mathrm{~Hz}) ; \\
1.69-1.56(\mathrm{~m}, 2 \mathrm{H} \mathrm{J}=7.34 \mathrm{~Hz})\end{array}$ & $0.91(\mathrm{t}, 3 \mathrm{H} \mathrm{J}=7.38 \mathrm{~Hz})$ & $3.84(\mathrm{~s}, 3 \mathrm{H})-\mathrm{OCH}_{3}$ \\
\hline 7 & 11.68 & 11.09 & 8.37 & $\begin{array}{l}7.40-7.21(\mathrm{~m}, 6 \mathrm{H}) \\
7.09-6.88(\mathrm{~m}, 3 \mathrm{H})\end{array}$ & $\begin{array}{l}5.45(\mathrm{~s}, 2 \mathrm{H}) ; 5.08(\mathrm{~s}, 2 \mathrm{H}) \\
2.68-2.55(\mathrm{t}, 2 \mathrm{H} \mathrm{J}=7.36 \mathrm{~Hz}) ; \\
1.68-1.55(\mathrm{~m}, 2 \mathrm{H} \mathrm{J}=7.38 \mathrm{~Hz})\end{array}$ & $0.92(\mathrm{t}, 3 \mathrm{H} \mathrm{J}=7.33 \mathrm{~Hz})$ & $\begin{array}{l}4.07(q, 2 \mathrm{H})-\mathrm{OCH}_{2} \\
1.32(\mathrm{t}, 3 \mathrm{H})-\mathrm{OCCH}_{3}\end{array}$ \\
\hline 8 & 12.03 & 11.12 & 8.43 & $\begin{array}{l}\text { 8.38-8.19 (d, 2H); } \\
\text { 8.02-7.88 (d, 2H); } \\
7.36-7.19(\mathrm{~m}, 5 \mathrm{H})\end{array}$ & $\begin{array}{l}5.54(\mathrm{~s}, 2 \mathrm{H}) ; 5.02(\mathrm{~s}, 2 \mathrm{H}) ; \\
2.67-2.59(\mathrm{t}, 2 \mathrm{H} \mathrm{J}=7.39 \mathrm{~Hz}) ; \\
1.73-1.59(\mathrm{~m}, 2 \mathrm{H} \mathrm{J}=7.36 \mathrm{~Hz})\end{array}$ & $0.90(\mathrm{t}, 3 \mathrm{H} \mathrm{J}=7.38 \mathrm{~Hz})$ & - \\
\hline 9 & 11.43 & 11.09 & 7.85 & $\begin{array}{l}7.54-7.41(\mathrm{~d}, 2 \mathrm{H}) \\
7.38-7.19(\mathrm{~m}, 5 \mathrm{H}) \\
6.76-6.63(\mathrm{~d}, 2 \mathrm{H})\end{array}$ & $\begin{array}{l}5.42(\mathrm{~s}, 2 \mathrm{H}) ; 5.06(\mathrm{~s}, 2 \mathrm{H}) ; \\
2.69-2.56(\mathrm{t}, 2 \mathrm{H} \mathrm{J}=7.43 \mathrm{~Hz}) ; \\
1.74-1.58(\mathrm{~m}, 2 \mathrm{H} \mathrm{J}=7.39 \mathrm{~Hz})\end{array}$ & $0.90(\mathrm{t}, 3 \mathrm{H} \mathrm{J}=7.37 \mathrm{~Hz})$ & $\begin{array}{l}2.99-2.87(\mathrm{~s}, 6 \mathrm{H})- \\
\mathrm{N}-\mathrm{CH}_{3}\end{array}$ \\
\hline 10 & 11.56 & 11.01 & 7.89 & $\begin{array}{l}7.38-7.17(\mathrm{~m}, 5 \mathrm{H}) \\
6.82-6.75(\mathrm{~m}, 3 \mathrm{H})\end{array}$ & $\begin{array}{l}5.44(\mathrm{~s}, 2 \mathrm{H}) ; 5.07(\mathrm{~s}, 2 \mathrm{H}) ; \\
2.69-2.54(\mathrm{t}, 2 \mathrm{H} \mathrm{J}=7.36 \mathrm{~Hz}) ; \\
1.72-1.60(\mathrm{~m}, 2 \mathrm{H} \mathrm{J}=7.36 \mathrm{~Hz})\end{array}$ & $0.92(\mathrm{t}, 3 \mathrm{H} \mathrm{J}=7.36 \mathrm{~Hz})$ & $3.74(\mathrm{~s}, 3 \mathrm{H})-\mathrm{OCH}_{3}$ \\
\hline 11 & 11.69 & 11.09 & 8.30 & $\begin{array}{l}7.38-7.19(\mathrm{~m}, 5 \mathrm{H}) ; \\
7.12-7.03(\mathrm{~m}, 3 \mathrm{H})\end{array}$ & $\begin{array}{l}5.47(\mathrm{~s}, 2 \mathrm{H}) ; 5.06(\mathrm{~s}, 2 \mathrm{H}) ; \\
2.66-2.58(\mathrm{t}, 2 \mathrm{H} \mathrm{J}=7.42 \mathrm{~Hz}) ; \\
1.72-1.59(\mathrm{~m}, 2 \mathrm{H} \mathrm{J}=7.35 \mathrm{~Hz})\end{array}$ & $0.90(\mathrm{t}, 3 \mathrm{H} \mathrm{J}=7.38 \mathrm{~Hz})$ & $\begin{array}{l}3.82(\mathrm{~s}, 3 \mathrm{H})-\mathrm{OCH}_{3} \\
3.72(\mathrm{~s}, 3 \mathrm{H})-\mathrm{OCH}_{3}\end{array}$ \\
\hline 12 & 11.67 & 11.14 & 7.88 & $7.42-7.10(\mathrm{~m}, 5 \mathrm{H})$ & $\begin{array}{l}5.41(\mathrm{~s}, 2 \mathrm{H}) ; 5.07(\mathrm{~s}, 2 \mathrm{H}) ; \\
2.68-2.59(\mathrm{t}, 2 \mathrm{H} \mathrm{J}=7.36 \mathrm{~Hz}) ; \\
1.71-1.59(\mathrm{~m}, 2 \mathrm{H} \mathrm{J}=7.34 \mathrm{~Hz})\end{array}$ & $\begin{array}{l}3.71(\mathrm{~s}, 3 \mathrm{H}) \\
0.91(\mathrm{t}, 3 \mathrm{H} \mathrm{J}=7.32 \mathrm{~Hz})\end{array}$ & $2.58(\mathrm{~s}, 3 \mathrm{H})-\mathrm{N}-\mathrm{CH}_{3}$ \\
\hline 13 & 11.69 & 11.11 & 7.86 & 7.4-7.16 (m, 5H) & $\begin{array}{l}5.43(\mathrm{~s}, 2 \mathrm{H}) ; 5.05(\mathrm{~s}, 2 \mathrm{H}) \\
2.67-2.57(\mathrm{t}, 2 \mathrm{H} \mathrm{J}=7.37 \mathrm{~Hz}) ; \\
1.72-1.61(\mathrm{~m}, 2 \mathrm{H} \mathrm{J}=7.31 \mathrm{~Hz})\end{array}$ & $\begin{array}{l}3.76 ;(\mathrm{s}, 3 \mathrm{H}) ; \\
0.92(\mathrm{t}, 3 \mathrm{H} \mathrm{J}=7.33 \mathrm{~Hz})\end{array}$ & $\begin{array}{l}2.77(q, 2 \mathrm{H})-\mathrm{N}-\mathrm{CH}_{2} ; \\
1.15(3 \mathrm{H} . \mathrm{T})-\mathrm{N}-\mathrm{C}-\mathrm{CH}_{3}\end{array}$ \\
\hline 14 & 11.32 & 11.14 & - & $\begin{array}{l}7.61-7.50 \text { (1Н. д); } \\
\text { 7.48-7.19 (6Н. м); } \\
7.17-6.92(1 \mathrm{H} . \text { т); } \\
6.91-6.83 \text { (1Н. д) }\end{array}$ & $\begin{array}{l}5.37(\mathrm{~s}, 2 \mathrm{H}) ; 5.09(\mathrm{~s}, 2 \mathrm{H}) ; \\
2.66-2.57(\mathrm{t}, 2 \mathrm{H} \mathrm{J}=7.34 \mathrm{~Hz}) ; \\
1.73-1.63(\mathrm{~m}, 2 \mathrm{H} \mathrm{J}=7.37 \mathrm{~Hz})\end{array}$ & $0.91(\mathrm{t}, 3 \mathrm{H} \mathrm{J}=7.39 \mathrm{~Hz})$ & $12.72(\mathrm{~s}, 1 \mathrm{H})-\mathrm{NH}$ \\
\hline 15 & 12.08 & 11.15 & 8.61 & $\begin{array}{l}\text { 8.45 (s, 1H); } \\
\text { 8.1-8.06 (d, 1H); } \\
\text { 8.01-7.74 (m, 2H); } \\
\text { 7.71-7.54 (d, 1H); } \\
\text { 7.39-7.16 (m, 5H) }\end{array}$ & $\begin{array}{l}5.49(\mathrm{~s}, 2 \mathrm{H}) ; 5.08(\mathrm{~s}, 2 \mathrm{H}) ; \\
2.65-2.54(\mathrm{t}, 2 \mathrm{H} \mathrm{J}=7.36 \mathrm{~Hz}) ; \\
1.71-1.61(\mathrm{~m}, 2 \mathrm{H} \mathrm{J}=7.33 \mathrm{~Hz})\end{array}$ & $0.91(\mathrm{t}, 3 \mathrm{H} \mathrm{J}=7.32 \mathrm{~Hz})$ & - \\
\hline 16 & 11.87 & 11.02 & - & $\begin{array}{l}\text { 7.97-7.83 (d, 2H); } \\
7.49-7.41(\mathrm{~d}, 2 \mathrm{H}) \\
7.37-7.19(\mathrm{~m}, 5 \mathrm{H})\end{array}$ & $\begin{array}{l}5.52(\mathrm{~s}, 2 \mathrm{H}) ; 5.08(\mathrm{~s}, 2 \mathrm{H}) ; \\
2.67-2.57(\mathrm{t}, 2 \mathrm{H} \mathrm{J}=7.44 \mathrm{~Hz}) ; \\
1.72-1.61(\mathrm{~m}, 2 \mathrm{H} \mathrm{J}=7.42 \mathrm{~Hz})\end{array}$ & $\begin{array}{l}2.25(\mathrm{~s}, 3 \mathrm{H}) ; \\
0.91(\mathrm{t}, 3 \mathrm{H} \mathrm{J}=7.41 \mathrm{~Hz})\end{array}$ & - \\
\hline 17 & 11.08 & 10.86 & - & $\begin{array}{l}7.81-7.68(\mathrm{~d}, 2 \mathrm{H}) \\
7.40-7.19(\mathrm{~m}, 5 \mathrm{H}) \\
6.9-6.91(\mathrm{~d}, 2 \mathrm{H})\end{array}$ & $\begin{array}{l}5.51(\mathrm{~s}, 2 \mathrm{H}) ; 5.09(\mathrm{~s}, 2 \mathrm{H}) \\
2.67-2.56(\mathrm{t}, 2 \mathrm{H} \mathrm{J}=7.43 \mathrm{~Hz}) ; \\
1.72-1.59(\mathrm{~m}, 2 \mathrm{H} \mathrm{J}=7.38 \mathrm{~Hz})\end{array}$ & $\begin{array}{l}2.23(\mathrm{~s}, 3 \mathrm{H}) ; \\
0.91(\mathrm{t}, 3 \mathrm{H} \mathrm{J}=7.39 \mathrm{~Hz})\end{array}$ & - \\
\hline 18 & 11.12 & 10.75 & - & $\begin{array}{l}7.52-7.46(\mathrm{~d}, 2 \mathrm{H}) \\
7.41-7.14(\mathrm{~m}, 5 \mathrm{H}) \\
6.59-6.52(\mathrm{~d}, 2 \mathrm{H})\end{array}$ & $\begin{array}{l}5.49(\mathrm{~s}, 2 \mathrm{H}) ; 5.07(\mathrm{~s}, 2 \mathrm{H}) ; \\
2.68-2.57(\mathrm{t}, 2 \mathrm{H} \mathrm{J}=7.36 \mathrm{~Hz}) ; \\
1.72-1.60(\mathrm{~m}, 2 \mathrm{H} \mathrm{J}=7.37 \mathrm{~Hz})\end{array}$ & $\begin{array}{l}2.26(\mathrm{~s}, 3 \mathrm{H}) ; \\
0.91(\mathrm{t}, 3 \mathrm{H} \mathrm{J}=7.31 \mathrm{~Hz})\end{array}$ & - \\
\hline
\end{tabular}


and the substituent in the third position were resonated as two multiplets at $\delta 7.71-7.61 \mathrm{ppm}(\mathrm{m}, 4 \mathrm{H})$ and 7.27-7.19 ppm (m, $5 \mathrm{H})$ respectively. Methylene protons were registered as singlets for substituents in positions 7 (5.48 ppm s, 2H) and 3 (5.06 ppm, s, 2H) of xanthine moiety, and triplet (2.67-2.58 ppm, $\mathrm{t}, 2 \mathrm{H})$ and multiplet $(1.74-1.58 \mathrm{ppm}, \mathrm{m}, 2 \mathrm{H})$ for the propyl radical at $\mathrm{C} 8$. The presence of the $\mathrm{CH}_{3}$ group was confirmed by a three-proton triplet at $0.92 \mathrm{ppm}(\mathrm{s}, 3 \mathrm{H})$.

\section{Discussion}

Biological activity of synthesized (3-benzyl-8-propylxanthin-7-yl)acetohydrazide derivatives was predicted by using PASS software [9]. All the compounds showed a good probability of antimicrobial activity (compounds 4-6, 8-10, 14), which makes a good foundation for the in-depth investigation for compounds with high antimicrobial activity. The results of the study of antibacterial and fungicidal activities were described in our previous work [10].

The reason for an in-depth study of the diuretic activity of the synthesized compounds was based on a molecular docking investigation. Interaction of (3-benzyl-8-propylxanthin-7-yl) acetohydrazide (1) and its derivatives $(2-5,7-9,12-14,16,17)$ with the $\mathrm{A}_{1}$ adenosine receptor has resulted in high affinity values. This result confirms the trend discovered in earlier in vitro study of diuretic activity of the compound 1 [11]. In conclusion, derivatives of (3-benzyl-8-propylxanthin-7-yl)acetohydrazide could be a promising starting material for further structural optimization to obtain new and more potent diuretic agents [12].

\section{Conclusions}

1. Preparative methods for the synthesis of the novel (3-benzyl-8-propylxanthin-7-yl)acetohydrazide and its derivatives have been developed.

2. The structures of the synthesized compounds was confirmed by various physical-chemical methods.

3. Within the investigated substances we identified compounds displayed good antimicrobial and diuretic activity.

\section{Funding}

The research is carried out within the RDW of Zaporizhzhia State Medical University: "Synthesis, physicochemical properties of N-substitued purines, xanthines and its heteroannelated derivatives", state registration number $0110 \mathrm{U} 000908$.

Conflicts of interest: authors have no conflict of interest to declare. Конфлікт інтересів: відсутній.

\section{Information about authors:}

Aleksandrova K. V., PhD, DSc, Professor, Head of the Department of Biochemistry, Zaporizhzhia State Medical University, Ukraine. ORCID ID: 0000-0003-4436-4036

Mykhalchenko Ye. K., PhD, Assistant of the Department of Biochemistry, Zaporizhzhia State Medical University, Ukraine.

ORCID ID: 0000-0002-6388-2966

Shkoda O. S., PhD, Associated Professor of the Department of Biochemistry, Zaporizhzhia State Medical University, Ukraine. ORCID ID: 0000-0002-5678-2801
Vasyliev D. A., PhD, Senior teacher of the Department of Biochemistry, Zaporizhzhia State Medical University, Ukraine.

ORCID ID: 0000-0002-9671-1397

\section{Відомості про авторів:}

Александрова К. В., д-р хім. наук, професор, зав. каф. біологічної хімії, Запорізький державний медичний університет, Україна.

Михальченко Є. К., PhD, асистент каф. біологічної хімії, Запорізький державний медичний університет, Україна.

Шкода О. C., PhD, доцент каф. біологічної хімії, Запорізький державний медичний університет, Україна.

Васильєв Д. A., PhD, старший викладач каф. біологічної хімії, Запорізький державний медичний університет, Україна

\section{Сведения об авторах:}

Александрова Е. В., д-р хим. наук, профессор, зав. каф.

биологической химии, Запорожский государственный медицинский университет, Украина.

Михальченко E. К., PhD, ассистент каф. биологической химии,

Запорожский государственный медицинский университет, Украина. Шкода A. C., PhD, доцент каф. биологической химии, Запорожский государственный медицинский университет, Украина.

Васильев Д. А., PhD, старший преподаватель каф. биологической химии, Запорожский государственный медицинский университет, Украина.

\section{References}

[1] Ojha, R., Singh, J., Ojha, A., Singh, H., Sharma, S., \& Nepali, K. (2017). An updated patent review: xanthine oxidase inhibitors for the treatment of hyperuricemia and gout (2011-2015). Expert opinion on therapeutic patents, 27(3), 311-345. https://doi.org/10.1080/13543776.2017.1261111

[2] Marx, D., Schnakenburg, G., Grimme, S., \& Müller, C. E. (2019). Structural and Conformational Studies on Carboxamides of 5,6-Diaminouracils-Precursors of Biologically Active Xanthine Derivatives. Molecules, 24(11), 2168. https://doi.org/10.3390/molecules24112168

[3] Marx, D., Wingen, L. M., Schnakenburg, G., Müller, C. E., \& Scholz, M. S. (2019). Fast, Efficient, and Versatile Synthesis of 6-amino-5-carboxamidouracils as Precursors for 8-Substituted Xanthines. Frontiers in chemistry, 7, 56. https://doi.org/10.3389/fchem.2019.00056

[4] Mikhal'chenko, E. K., Aleksandrova, K. V., \& Levich S. V. (2016). Syntez i fizyko-khimichni vlastyvosti 3-benzyl-8-propilksantynu ta yoho vodorozchynnykh solei [Synthesis and physical-chemical properties of 3-benzyl-8-propylxanthine and its water-soluble salts]. Current issues in pharmacy and medicine: science and practice, (1), 26-30. https://doi.org/10.14739/24092932.2016.1.62005

[5] Aleksandrova, K. V., Levich, S. V., Belenichev, I. F., \& Shkoda, A. S. (2015) Research of energotropic properties of 3-benzylxanthine derivative-prospective neuroprotector. International Journal of Pharmacy, 5(1), 1-4.

[6] Suravajhala, R., Poddar, R., Nallapeta, S, \& Ullah, S. (2014). Xanthine Derivatives: A Molecular Modeling Perspective. In P. B. Kavi Kishor, R. Bandopadhyay, P. Suravajhala (Eds.), Agricultural Bioinformatics (pp. 283-291). Springer, New Delhi. https://doi.org/10.1007/978-81-322-1880-7 17

[7] Monteiro, J. P., Alves, M. G., Oliveira, P. F., \& Silva, B. M. (2016). Structure-Bioactivity Relationships of Methylxanthines: Trying to Make Sense of All the Promises and the Drawbacks. Molecules, 21(8), 974. https://doi. org/10.3390/molecules21080974

[8] Levich, S. V., Shkoda, O. S., Kamyshnyi, O. M., Aleksandrova, K. V., \& Polischuk, N. M. (2013). 2-(3-benzyl-8-metylksantyn-7-il)-N-[(1E,2E)-3-(5-nitrofuran-2-il)prop-2-en-1-iliden]-atsetohid-razyd, yakyi vyiavliaie antymikrobnu diiu [2-(3-benzyl-8-methylxanthine-7-yl)-N >-[(1E,2E)-3-(5-nitrofuran-2-YL) prop-2-ene-1-yliden]-acetohydrazide exhibiting antimicrobial action]. Ukraine Patent UA 83840

[9] Aleksandrova, K. V., Mykhalchenko, Ye. K., \& Levich, S. V. (2017). Vyvchennia protymikrobnykh ta protyhrybkovykh vlastyvostei 7-zamishchenykh 3-benzyl-8-propilksantyniv [Study of antimicrobial and antifungal properties of 7-substituted 3-benzyl-8-propylxanthines]. Medychnyi forum, (10), 9-12. [in Ukrainian].

[10] Way2Drug. (n.d.). PASS Online. Way2Drug.com. http://www.pharmaexpert. ru/PASSOnline

[11] Aleksandrova, K. V., Mykhalchenko, Ye. K., Pruhlo, Ye. S., \& Sinchenko, D. M. (2018). Hidrazyd 3-benzyl-8-propilksantyn-7-il atsetatnoi kysloty, yakyi vyiavliaie diuretychnu diiu [3-Benzyl-8-propylxanthin-7-yl acetic acid hydrazide, which has a diuretic effect]. Ukraine Patent UA 128510. https://base.uipv.org/ searchINV/search.php?action=viewdetails\&IdClaim $=251158$

[12] Mykhalchenko, Ye., Aleksandrova, K., Shkoda, O., \& Pruglo, Ye. (2019). Prospective biological active compounds among 7-substituted of 3-benzyl-8-propylxanthines for treatment of metabolic syndrome pathologies. EUREKA: Health Sciences, (5), 52-58. https://doi.org/10.21303/2504-5679.2019.00988 